\title{
Effect of Attached Resonators on Magnon Propagation in Dipole-Coupled Nanostructured Waveguide
}

\author{
H. Al-Wahsh ${ }^{a, b, *}$, L. Dobrzyński ${ }^{a}$, A. Akjouj $^{a}$, \\ B. DJafari-Rouhani ${ }^{a}$ AND E.H. El Boudouti ${ }^{a, c}$
}

\author{
${ }^{a}$ Institut d'Electronique, de Microélectronique et de Nanotechnologie (IEMN), \\ UMR CNRS 8520, UFR de Physique, Université des Sciences et Technologies de Lille, \\ 59655 Villeneuve d'Ascq Cédex, France \\ ${ }^{b}$ Engineering Mathematics and Physics Department, Faculty of Engineering, \\ Benha University, 11629 Cairo, Egypt \\ ${ }^{c}$ LPMR, Département de Physique, Faculté des Sciences, \\ Université Mohamed I, 60000 Oujda, Morocco
}

Received: 11.06.2020 \& Accepted: 26.08.2020

\begin{abstract}
We report a theoretical study on the effect of the exchange coupling on magnon propagation in a waveguide, composed of a dipole-coupled nanometric magnetic cluster chain and a few additional clusters near the chain. We show that an appropriate choice of the exchange coupling constant $J$ or the geometrical/magnetic parameters of the structure can lead either to narrow dips or to narrow peaks in the transmission spectrum of magnons along the chain. The phase of the transmission amplitude, the state phase shift and the variation of the density of states are also discussed as a function of frequency for different values of $J$. The effect of attenuation on the transmission spectra is also discussed. The results are obtained by means of the Green's function technique. The presented study could be useful for constructing the selecting or rejecting magnon filters.
\end{abstract}

topics: magnon, transmission, filtering, nanostructure

\section{Introduction}

Magnonic crystals, i.e., magnetic structures for spin-wave propagation with a periodic modulation of structural, compositional or magnetic properties, are the counterpart of photonic and phononic crystals where the spin wave band structure consists of intervals of allowed frequencies and forbidden bands (gaps) in which there are no allowed magnonic states [1-4]. Magnonic crystals have become the focus of significant attention for their interesting advantages in comparison with their photonic and phononic crystals counterparts. Spin waves perhaps display more diversity of dispersion characteristics $[5,6]$ than any other kind of wave (e.g., electromagnetic or acoustic). The dispersion of the spin waves with a wavelength shorter than the period of a magnonic crystal contains magnonic band gaps, while in the case of spin waves with a longer wavelength, the same magnonic crystal will represent an effectively continuous medium with properties defined not only by those of the constituent magnetic materials but also by the details of the geometrical and micromagnetic structure [7-9]. Furthermore, spin waves enable easy tunability by an external magnetic field and reprogrammability of the band structure $[4,10,11]$. It is worth to mention that spin waves typically exist in the microwave frequency range and their wavelength can be decreased to the nanoscale, making them a promising prospect for the development of microwave information processing devices [5-8, 12].

Research in the area of nanofabrication technology and the production of high-performance magnetic materials have made possible the preparation of regular arrays of magnetic particles of different shapes and sizes [13-16]. For magnetic nanoclusters, interest has focused on the determination of their internal magnetic structure which is of fundamental importance for practical applications. In this direction, d'Albuquerque et al. proposed a method to investigate the phase diagrams of nanosized systems [17, 18]. Their method is based on a scaling relation when starting from the phase diagrams of much smaller systems. In [19], Rivkin et al. introduced the "discrete dipole approximation" method to calculate the modes along a linear chain of ferromagnetic particles having an arbitrary shape. They also extend their technique to treat infinite lattices of 
such nanoparticles [20, 21]. Let us also mention the method reported by Khater et al. to calculate the spin mode energies of a magnetic cluster on the surface of a magnetic substrate [22, 23]. They also investigated the presence of magnetic excitations localized on clusters supported on the surface of a magnetically ordered substrate. Spin wave excitations in dipole-coupled nanodisks from permalloy are investigated in [24], while in [25], spin wave excitations in two-dimensional magnetic nanodots in the vortex state are explored. On experimental grounds, magnetic force microscopy and magnetization measurements have been used to investigate the arrangement of the magnetic moments within the particles [16, 26, 27]. The magnetization and resonance frequencies of submicron Fe magnetic dot arrays were investigated by the Brillouin light scattering techniques and magneto-optic Kerr effects [28, 29]. In [30], Shimon et al. used the microfocused Brillouin light scattering spectroscopy to investigate the dynamic response of a single magnetic disk as a function of closely-spaced neighboring disks coupling configurations. They also developed a simple method to estimate the strength of the effective dipolar interaction based on experimental data fitting. The dependence of the magnetic moments of the free clusters on size and temperature were investigated using a SternGerlach magnet and a time-of-flight mass spectrometer [31]. The X-ray magnetic circular dichroism technique has been used to investigate the orbital and spin magnetic moments of supported clusters [32, 33]. The Brillouin scattering is extensively used to study spin excitations in magnetic systems including nanoarrays [34-36]. Using coherent inelastic light scattering from a microwave-excited array of magnetic particles, Giovannini et al. have shown that it should be possible to drive specific spin modes into nonlinear regimes [37].

Depending on the relative importance of the magnetic dipole-dipole and exchange interactions, different models for the magnetic behavior need to be employed. For instance, for small enough values of the excitation wave vector (typically less than about $10^{7} \mathrm{~m}^{-1}$ in a ferromagnet), dipolar effects are dominant for the dynamics and magnetostatic modes are the resulting excitations that propagate in such structures. On the other hand, at large enough wave vectors, typically $>10^{8} \mathrm{~m}^{-1}$, the exchange interaction, which thus provides the restoring force for spin waves, will be dominant. The magnon wave vector at which these two types of interaction are comparable may vary for different magnetic materials $[38,39]$. In this work, we adopt the "discrete dipole approximation" model [19, 40] for a ferromagnetic chain with additional clusters in order to discuss the intermediate (or "dipole-exchange") case, where both types of interactions influence the dynamical behavior. We restrict the exchange interaction to the nearest neighbors only. This is a first step towards more sophisticated calculations.

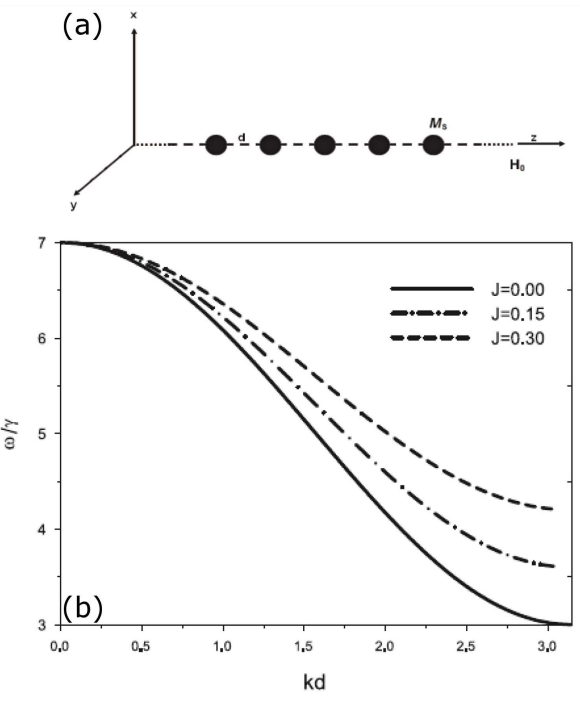

Fig. 1. (a) Infinite linear cluster chain. The distance between two neighboring clusters is $d$, the static magnetic moments is $\boldsymbol{M}_{s}$ and the external magnetic field is $\boldsymbol{H}_{0}$, along the $z$ axis. (b) Frequency as a function of the reduced wave vector $k d$ (dispersion relation (6)) for an infinite linear chain. The parameters are $\gamma=M_{s}=d=H_{0}=1$. The plots are given for $J=0$ (solid curve), $J=0.15$ (dashed-dotted curve) and $J=0.30$ (dashed curve), respectively.

In previous publications [41-42], we proposed a theory of magnon propagation in waveguides, composed of an infinite nanometric magnetic cluster chain and additional clusters near the chain. We have taken into account only the dipole-dipole interactions between the nearest-neighbor cluster local magnetic moments. It has been shown that the coupling of the infinite chain to the adsorbed clusters (which play the role of resonators) induces peaks and dips (or zeros) in the transmission spectrum. These resonant states result from an internal resonance of the structure when such a resonance is situated in the vicinity of a zero of transmission or placed between two zeros of transmission. Let us finally mention that in [43] we presented a multiplexing device that can transfer one magnon state from one cluster chain to the other, leaving all the other neighbor states unaffected.

Motivated by these findings and based on model calculations in which the strength of the exchange coupling constant $J$ has been manipulated, we present in this work a detailed analysis of various features induced by the exchange coupling inside the transmission band of a waveguide composed of an infinite nanometric ferromagnetic cluster chain (see Fig. 1a) and additional clusters near the chain (the geometry of the waveguide is depicted in Fig. 2). The distance between the two clusters in the infinite chain and in the three cluster chains is considered to be $d$ and the distance between the additional clusters and the wire is considered to be $d_{1}$. The nanoparticles in the infinite chain are to bear 
a permanent magnetic moment $\boldsymbol{M}_{s}$, while that of the three additional clusters is $\boldsymbol{M}_{s}^{\prime}$. We show analytically and numerically that an appropriate choice of the exchange coupling constant $J$ or the geometrical/magnetic parameters can lead either to narrow dips or to narrow peaks in the transmission spectrum of magnons along the ferromagnetic chain. We also study the transmission phase, the state phase shift and the variation of the density of states (VDOS). The results achieved in this work have been established through the analysis of the transmission function (amplitude and phase) obtained within the framework of the Green's function method.

This paper is organized as follows. In Sect. 2, we recall the expressions of dispersion, transmission and reflection coefficients, phase and VDOS associated to the structure depicted in Fig. 2. These results are necessary for the understanding of the new phenomenon obtained for the structure proposed in this work. Section 3 is devoted to the analysis and discussion of the results. Finally, conclusions are drawn in Sect. 4.

\section{Transmission and reflection coefficients}

Our calculation is performed with the help of the interface response theory of the discrete media. This theory allows the calculation of the Green's function of any composite material and then the transmission and reflection coefficients. In what follows, we shall avoid the details of these calculations which are similar to those given in [41-43]. Instead, we just give the expressions of the dispersion, transmission and reflection coefficients.

Following the "discrete dipole approximation" method [19-40], the magnon modes are assumed to follow the Landau-Lifshitz equation

$-\frac{1}{\gamma} \frac{\mathrm{d} \boldsymbol{m}_{i}}{\mathrm{~d} t}=\boldsymbol{m}_{i} \times \boldsymbol{h}_{i}^{\text {total }}+\frac{\beta}{M_{s}} \boldsymbol{m}_{i} \times\left(\boldsymbol{m}_{i} \times \boldsymbol{h}_{i}^{\text {total }}\right)$,

where $\boldsymbol{m}_{i}$ is the magnetic moment on cluster $i$, $\gamma$ and $M_{s}$, respectively, are the gyromagnetic ratio $[\mathrm{GHz} / \mathrm{T}]$ and saturation magnetization $\left[\mathrm{T} \mu \mathrm{m}^{3}\right]$, and $\beta$ is the dimensionless damping parameter. The total effective field $\boldsymbol{h}_{i}^{\text {total }}$ experienced by the $i$-th cluster is given by

$$
\boldsymbol{h}_{i}^{\text {total }}=\boldsymbol{h}_{i}^{\text {dipole }}+\boldsymbol{h}_{i}^{\text {exchange }}+\boldsymbol{H}_{0} .
$$

Each cluster feels the effect of the others through the dipole-dipole field

$$
\boldsymbol{h}_{i}^{\text {dipole }}=\sum_{i \neq l}\left(\frac{3 \boldsymbol{r}_{i l}\left(\boldsymbol{m}_{l} \cdot \boldsymbol{r}_{i l}\right)}{r_{i l}^{5}}-\frac{\boldsymbol{m}_{l}}{r_{i l}^{3}}\right)
$$

and the exchange field of the nearest-neighbor (NN) Heisenberg type

$$
\boldsymbol{h}_{i}^{\text {exchange }}=J \sum_{i}^{N N} \boldsymbol{m}_{i},
$$

where the exchange constant $J$ could be adjusted to reproduce the experimental continuum limit coefficient of the gradient energy [19, 44]. In addition, the clusters interact with the external magnetic field $\boldsymbol{H}_{0}$ and, if present, with an anisotropy field $\boldsymbol{h}_{i}^{\text {anisotropy }}$ (in this work it will be neglected). We linearize (1) by writing $\boldsymbol{m}_{i}=\boldsymbol{m}_{i}^{(0)}+\boldsymbol{m}_{i}^{(1)}$, where the $\boldsymbol{m}_{i}^{(0)}$ have only one non-zero component due to the static magnetic moment $\boldsymbol{M}_{\boldsymbol{s}}$ (for the chain clusters) and $\boldsymbol{M}_{s}^{\prime}$ (for the three additional clusters) along the $z$ axis. The $\boldsymbol{m}_{i}^{(1)}$ has only nonzero components $m_{i x}$ and $m_{i y}$ along the $x$ - and $y$ axis. Inserting these expressions into (1) and retaining only the first-order terms, one yields

$$
\begin{aligned}
& -\frac{1}{\gamma} \frac{\mathrm{d} \boldsymbol{m}_{i}^{(1)}}{\mathrm{d} t}=\boldsymbol{m}_{i}^{(0)} \times \boldsymbol{h}_{i}^{(1)}+\boldsymbol{m}_{i}^{(1)} \times \boldsymbol{h}_{i}^{(0)} \\
& \quad+\boldsymbol{m}_{i}^{(1)} \times \boldsymbol{H}_{0}+\frac{\beta}{M_{s}} \boldsymbol{m}_{i}^{(0)} \\
& \quad \times\left[\boldsymbol{m}_{i}^{(0)} \times \boldsymbol{h}_{i}^{(1)}+\boldsymbol{m}_{i}^{(1)} \times \boldsymbol{h}_{i}^{(0)}+\boldsymbol{m}_{i}^{(1)} \times \boldsymbol{H}_{0}\right],
\end{aligned}
$$

where $\boldsymbol{h}_{i}^{(n)}=\boldsymbol{h}_{i}^{\operatorname{dipole}(n)}+\boldsymbol{h}_{i}^{\text {exchange }(n)}, n=0,1$.

\subsection{Dipolar-spin wave modes: periodic boundary conditions}

Now we turn to the calculation of the dipole spin waves for the case of chain clusters with the magnetic field parallel to the chain. We do this for the case of a chain with free ends and for the one satisfying periodic boundary conditions. In the coordinate system shown in Fig. 1a, all the spins lie along the $z$-axis. The component of the oscillation parallel to the applied field vanishes, so that the nonzero components of the oscillations are confined to the $x-y$ plane. Further, we assume solutions of the form $\boldsymbol{m}_{i}^{(1)}(t)=\boldsymbol{m}_{i}^{(1)} \exp (-\mathrm{i} \omega t)$. In the case of zero damping, (5) takes the following form

$$
\begin{aligned}
& \frac{\mathrm{i} \omega}{\gamma} m_{i x}^{(1)}=\frac{M_{s}}{d^{3}}\left(m_{(i+1) y}^{(1)}+m_{(i-1) y}^{(1)}\right) \\
& +m_{i y}^{(1)}\left(H_{0}+\frac{4 M_{s}}{d^{3}}\right) \\
& +J M_{s}\left(2 m_{i y}^{(1)}-m_{(i-1) y}^{(1)}-m_{(i+1) y}^{(1)}\right), \\
& \frac{\mathrm{i} \omega}{\gamma} m_{i y}^{(1)}=-\frac{M_{s}}{d^{3}}\left(m_{(i+1) x}^{(1)}+m_{(i-1) x}^{(1)}\right) \\
& -m_{i x}^{(1)}\left(H_{0}+\frac{4 M_{s}}{d^{3}}\right) \\
& -J M_{s}\left(2 m_{i x}^{(1)}-m_{(i-1) x}^{(1)}-m_{(i+1) x}^{(1)}\right) .
\end{aligned}
$$

The resulting coupled equations have the structure of a vector eigenvalue problem. It can be solved using the standard transformation $m_{i}^{+}=m_{i x}+\mathrm{i} m_{i y}$ and $m_{i}^{-}=m_{i x}-\mathrm{i} m_{i y}$ [18]. The eigenvalues give the frequencies of the (generally mixed) dipolar and/or spin-wave modes. For the infinite cluster chain, the proposed transformation enables to find a positive/negative-frequency solution. Namely, 


$$
\begin{aligned}
\omega & = \pm \gamma\left[H_{0}+\frac{2 M_{s}}{d^{3}}(2+\cos (k d))\right. \\
& \left.+2 M_{s} J(1-\cos (k d))\right], \\
\omega & \approx \pm \gamma\left[H_{0}+\frac{6 M_{s}}{d^{3}}+M_{s}\left(J d^{2}-\frac{1}{d}\right) k^{2}\right],
\end{aligned}
$$

where $k$ is the wave vector. Setting the exchange constant $J=0$, the problem is reduced then to purely Walker modes [45].

\subsection{Green's function results}

The corresponding Green's function elements associated with $m_{i}^{ \pm}$and the respectively negative $(+)$ and positive $(-)$ frequency bands are [46]:

$$
G_{ \pm}\left(n, n^{\prime}\right)=\frac{t_{ \pm}^{\left|n-n^{\prime}\right|}}{F_{ \pm}}
$$

where

$$
\begin{aligned}
& t_{ \pm}= \begin{cases}\zeta_{ \pm}-\sqrt{\zeta_{ \pm}^{2}-1}, & \zeta_{ \pm}>1 \\
\zeta_{ \pm} \pm \mathrm{i} \sqrt{1-\zeta_{ \pm}^{2}}, & -1<\zeta_{ \pm}<1, \\
\zeta_{ \pm}+\sqrt{\zeta_{ \pm}^{2}-1}, & \zeta_{ \pm}<-1,\end{cases} \\
& F_{ \pm}= \pm M_{S}\left(d^{-3}-J\right)\left(t_{ \pm}-t_{ \pm}^{-1}\right),
\end{aligned}
$$
and

$$
\zeta_{ \pm}=\frac{\mp\left(\frac{\omega}{\gamma} \pm H_{0}\right)-2 M_{s}\left(\frac{2}{d^{3}}+J\right)}{2 M_{s}\left(\frac{1}{d^{3}}-J\right)} .
$$

Consider now as the reference system the infinite cluster chain and the three additional clusters having a magnetic moment $\boldsymbol{M}_{s}^{\prime}$ and situated like depicted in Fig. 2 but without any interactions between themselves, nor with the chain clusters 1 , 2,3 in front of which they are deposited. When a progressive wave is launched from the left along the infinite chain, the relation of the transmission (reflection) function to the pulsation of the magnon signal $\omega[\mathrm{GHz}]$ can be derived using the Green's function method [47]. Let us write the transmission function $t(\omega)$ in the complex form as $t(\omega)=a+\mathrm{i} b=$ $\sqrt{T} \mathrm{e}^{\mathrm{i} \varphi}$, where $T$ is the transmission coefficient and $\varphi=\arctan (b / a)$ is the phase associated with the transmission field. Then, the transmission $T$ and reflection $R$ coefficients can be expressed as [47]:

$$
\begin{aligned}
& T=|t|^{2}=\left|F_{-} g\left(1_{-}, 3_{-}\right)\right|^{2}, \\
& R=|r|^{2}=\left|F_{-} g\left(1_{-}, 1_{-}\right)-1\right|^{2} .
\end{aligned}
$$

Here, one can easily deduce the conservation energy, namely $T+R=1$. Above, $g\left(1_{-}, 3_{-}\right)$and $g\left(1_{-}, 1_{-}\right)$are the Green's function elements between clusters 1 and 3 and on the cluster 1 for the $m_{i}^{-}$magnetic moment. The Green's function elements can be obtained from the inverse of the ma$\operatorname{trix}[g(M M)]^{-1}[48]$, i.e.,

$$
[g(M M)]^{-1}=[G(M M)]^{-1}+V_{I}(M M),
$$

where $G(M M)$ is the matrix of the Green's function elements in the interface cluster space $M(=$ $1,2,3,11,21,31)$. Its rows and columns are labeled by $\left(m_{1}^{+}, m_{2}^{+}, m_{3}^{+}, \quad m_{1}^{-}, m_{2}^{-}, m_{3}^{-}, \quad m_{11}^{+}, m_{21}^{+}, m_{31}^{+}\right.$, $\left.m_{11}^{-}, m_{21}^{-}, m_{31}^{-}\right)$. When switching on the dipoledipole and exchange interactions between the three additional clusters themselves and their nearestneighbors in the chain, the six clusters become perturbed by these interactions. But since two unknown ones, $m_{i}^{+}$and $m_{i}^{-}$, are attached to each cluster, it results in the twelve unknowns, i.e., to the interface space of dimension twelve and to the $12 \times 12$ interaction matrix $V_{I}(M M)[41,47]$. It is worth mentioning that the minimum number of perturbing clusters to ensure the effects studied in this work is three (for details see [41]). For the structure shown in Fig. 2, $[G(M M)]^{-1}$ and $V_{I}(M M)$ are given by

$$
[G(M M)]^{-1}=\left(\begin{array}{cccccccccccc}
Y_{+} & Z_{+} & 0 & 0 & 0 & 0 & 0 & 0 & 0 & 0 & 0 & 0 \\
Z_{+} & T_{+} & Z_{+} & 0 & 0 & 0 & 0 & 0 & 0 & 0 & 0 & 0 \\
0 & Z_{+} & Y_{+} & 0 & 0 & 0 & 0 & 0 & 0 & 0 & 0 & 0 \\
0 & 0 & 0 & Y_{-} & Z_{-} & 0 & 0 & 0 & 0 & 0 & 0 & 0 \\
0 & 0 & 0 & Z_{-} & T_{-} & Z_{-} & 0 & 0 & 0 & 0 & 0 & 0 \\
0 & 0 & 0 & 0 & Z_{-} & Y_{-} & 0 & 0 & 0 & 0 & 0 & 0 \\
0 & 0 & 0 & 0 & 0 & 0 & \Omega_{+} & 0 & 0 & 0 & 0 & 0 \\
0 & 0 & 0 & 0 & 0 & 0 & 0 & \Omega_{+} & 0 & 0 & 0 & 0 \\
0 & 0 & 0 & 0 & 0 & 0 & 0 & 0 & \Omega_{+} & 0 & 0 & 0 \\
0 & 0 & 0 & 0 & 0 & 0 & 0 & 0 & 0 & \Omega_{-} & 0 & 0 \\
0 & 0 & 0 & 0 & 0 & 0 & 0 & 0 & 0 & 0 & \Omega_{-} & 0 \\
0 & 0 & 0 & 0 & 0 & 0 & 0 & 0 & 0 & 0 & 0 & \Omega_{-}
\end{array}\right)
$$

where

$$
\begin{aligned}
& Y_{+}=\frac{F_{+}}{1-t_{+}^{2}}, \quad Y_{-}=\frac{F_{-}}{1-t_{-}^{2}}, \quad Z_{+}=-Y_{+} t_{+}, \quad Z_{-}=-Y_{-} t_{-} \quad T_{+}=Y_{+}\left(1+t_{+}^{2}\right), \\
& T_{-}=Y_{+}\left(1+t_{+}^{2}\right), \quad \Omega_{+}=\frac{\omega}{\gamma}+H_{0}, \quad \Omega_{-}=\frac{\omega}{\gamma}-H_{0} .
\end{aligned}
$$




$$
V_{I}(M M)=\left(\begin{array}{cccccccccccc}
A & 0 & 0 & 0 & 0 & 0 & -P & 0 & 0 & -Q & 0 & 0 \\
0 & A & 0 & 0 & 0 & 0 & 0 & -P & 0 & 0 & -Q & 0 \\
0 & 0 & A & 0 & 0 & 0 & 0 & 0 & -P & 0 & 0 & -Q \\
0 & 0 & 0 & -A & 0 & 0 & Q & 0 & 0 & P & 0 & 0 \\
0 & 0 & 0 & 0 & -A & 0 & 0 & Q & 0 & 0 & P & 0 \\
0 & 0 & 0 & 0 & 0 & -A & 0 & 0 & Q & 0 & 0 & P \\
-R & 0 & 0 & -S & 0 & 0 & B & D & 0 & 0 & 0 & 0 \\
0 & -R & 0 & 0 & -S & 0 & D & C & D & 0 & 0 & 0 \\
0 & 0 & -R & 0 & 0 & -S & 0 & D & B & 0 & 0 & 0 \\
S & 0 & 0 & R & 0 & 0 & 0 & 0 & 0 & -B & -D & 0 \\
0 & S & 0 & 0 & R & 0 & 0 & 0 & 0 & -D & -C & -D \\
0 & 0 & S & 0 & 0 & R & 0 & 0 & 0 & 0 & -D & -B
\end{array}\right)
$$

where

$$
\begin{aligned}
& A=M_{s}^{\prime}\left(J-\frac{1}{d_{1}^{3}}\right), \quad B=J\left(M_{s}+M_{s}^{\prime}\right)+\frac{2 M_{s}^{\prime}}{d^{3}}-\frac{M_{s}}{d_{1}^{3}}, \quad C=J\left(M_{s}+2 M_{s}^{\prime}\right)+\frac{4 M_{s}^{\prime}}{d^{3}}-\frac{M_{s}}{d_{1}^{3}} \\
& D=M_{s}^{\prime}\left(\frac{1}{d^{3}}-J\right), \quad P=M_{s}\left(J+\frac{1}{2 d_{1}^{3}}\right), \quad Q=\frac{3 M_{s}}{2 d_{1}^{3}}, \quad R=M_{s}^{\prime}\left(J+\frac{1}{2 d_{1}^{3}}\right), \quad S=\frac{3 M_{s}^{\prime}}{2 d_{1}^{3}} .
\end{aligned}
$$

\section{Applications and discussion of results}

We now turn to discussing our numerical results on dispersion, transmission, reflection and phase of transmission. In what follows, we illustrate the above results by assuming for simplicity $d=1 \mu \mathrm{m}[19,20,49], H_{0}=1 \mathrm{~T}$ and $M_{s}=1 \mathrm{~T} \mu \mathrm{m}^{3}$. We also limit ourselves to the case of identical exchange coupling between any two neighboring clusters in the structure. The unit of exchange constant $J$ is $\mu \mathrm{m}^{-3}$. Figure $1 \mathrm{~b}$ gives $\omega / \gamma$ versus the reduced wave vector $k d$ (for positive $k d$ only), for the infinite cluster chain without three additional clusters. The following values are shown: $J=0$ (solid curve), $J=0.15$ (dashed-dotted curve) and $J=0.30$ (dashed curve). One can notice that the width of the transmission band decreases with increasing the $J$ value. Furthermore, with the help of (8), one can see that the quantity $\omega / \gamma-H_{0}$ (for the assumed parameters) varies from $2(1+2 J)$ to 6 . This result confirms that the lower border of the transmission band is a function of $J$.

Figure 3a shows the frequency dependence of the transmission factor $T=|t|^{2}$, for $d_{1}=1.5 \mu \mathrm{m}$ and $M_{s}^{\prime}=1 \mathrm{~T} \mu \mathrm{m}^{3}$. A sharp zero of transmission appears at frequency $\left(\omega_{0}\right)$, a little above the middle of the bulk band. Such a narrow stop band could be useful for constructing a rejecting signal device. Figure $3 \mathrm{c}$ shows the variation of the phase as a function of $\frac{\omega}{\gamma}$ for the same parameters given in Fig. 3a. The variation of the phase shows an abrupt change in $\pi$ at the transmission zero induced by the additional clusters. The geometrical origin of this phase jump can clearly be seen in Fig. 3b which shows the frequency evolution of the real and imaginary parts of the transmission function $t(\omega)$ for $J=0$. This result demonstrates that the evolution has a loop-like behavior in the vicinity of the transmission zero. Furthermore, at the origin of

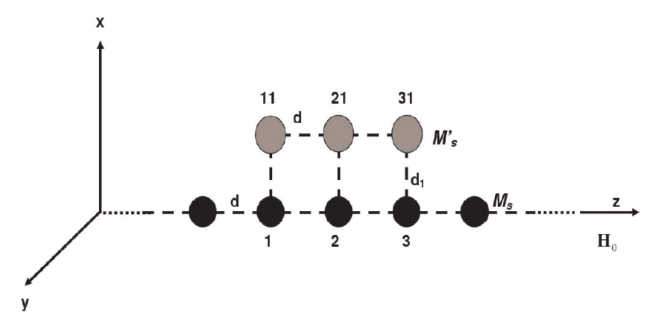

Fig. 2. Geometry of the considered nanometric device. It consists of one cluster chain and three attached clusters. The distances between these clusters are respectively $d$ and $d_{1}$, as indicated in the figure. The static magnetic moments are $\boldsymbol{M}_{s}$ (for the chain clusters) and $\boldsymbol{M}_{s}^{\prime}$ (for the three additional clusters) and the external magnetic field $\boldsymbol{H}_{0}$ is oriented along the waveguide ( $z$ axis).

the coordinates $(\operatorname{Re}(t)=0, \operatorname{Im}(t)=0)$, one can easily see that the value of the transmission function $\left.t(\omega)\right|_{\omega_{0}}=0$. This result agrees with the general statement given in $[50,51]$ that a sharp jump of the phase by $\pi$ does appear when the transmission function intersects the origin.

In addition to the information that can be deduced from the amplitude and the phase of the transmission function, one can also use the VDOS or the difference in the DOS due to the presence of a scatterer. This quantity is given by [52]:

$$
\Delta n(\omega)=\frac{1}{\pi} \frac{\mathrm{d} \eta(\omega)}{\mathrm{d} \omega}
$$

where

$$
\eta(\omega)=-\arg \left(\operatorname{Det}\left((g(M M))^{-1} G(M M)\right)\right)
$$

is the state phase shift. The derivative of this state phase has, within the bulk bands, the same value as the derivatives of the Friedel phase $[53,54]$ and the transmission phase. This state phase also includes 

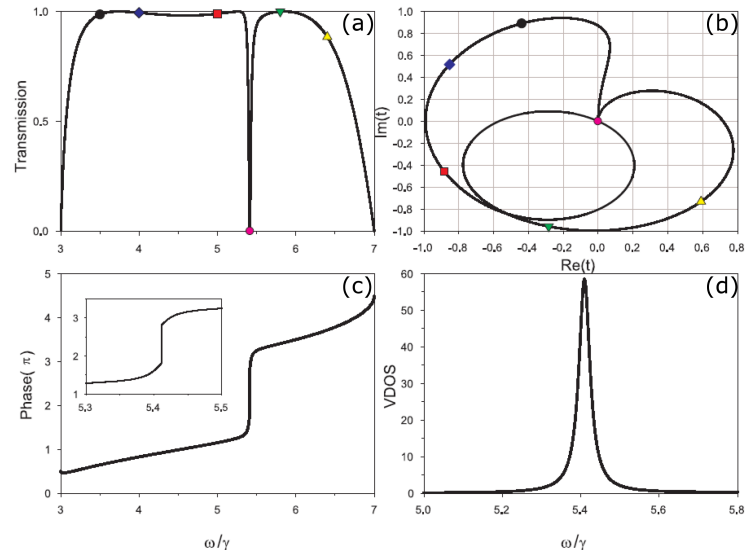

Fig. 3. (a) Transmission as a function of $\omega / \gamma$ for $H_{0}=1, M_{s}=M_{s}^{\prime}=1, d=1, d_{1}=1.5$ and $J=0.0$. (b) Evolution of real and imaginary parts of the transmission function $t(\omega)$. The parameters have the same values as in (a). (c) The same as in (a) but for the variation of the transmission phase.

(d) VDOS versus $\omega / \gamma$.

the $+\pi$ or $-\pi$ discontinuities due to the discrete states and to the localized states of the reference and final systems, and enables then to check the state conservation between these two systems. The Friedel phase is indirectly related to these discontinuities, when going to the Friedel sum rule, enabling to check - in particular for electrons the charge conservation. It is worth to note that the difference between the Friedel phase and the phase of the transmission amplitude has been reported in several works devoted to electronic transport in mesoscopic structures using the AharonovBohm systems [53, 55]. These studies are related to the investigation of the electronic states of quantum dots as well as to the understanding $[53,55,56]$ of the transmission phase jumps by $\pi$ between two adjacent resonances in relation to the experiments of Yacoby et al. [57, 58]. An experimental confirmation of this analogy has been performed by some of us $[59,60]$ for electromagnetic waves in coaxial cables. The analogy between the scattering properties of electrons, phonons, photons and magnons suggests that this type of feature can also appear in magnonic systems [61]. However, to our knowledge, such a study has not been performed yet in magnonic crystals.

Figure $3 \mathrm{~d}$ gives the variation between the final and reference systems of the VDOS versus the frequency. This quantity shows the existence of a resonance, blocking the transmission, at the frequency of the zero of transmission, for which $g\left(1_{-}, 3_{-}\right)$vanishes, see (14). The system acts as a rejecting filter. At this frequency, $g\left(1_{-}, 2_{-}\right)$vanishes too. Clusters 1, 11, 21 and 31 respond to an incident wave on cluster 1 but clusters 2 and 3 do not, so the wave is reflected. This response of clusters 11, 21 and 31 is consistent with the positive VDOS shown in Fig. 3d.

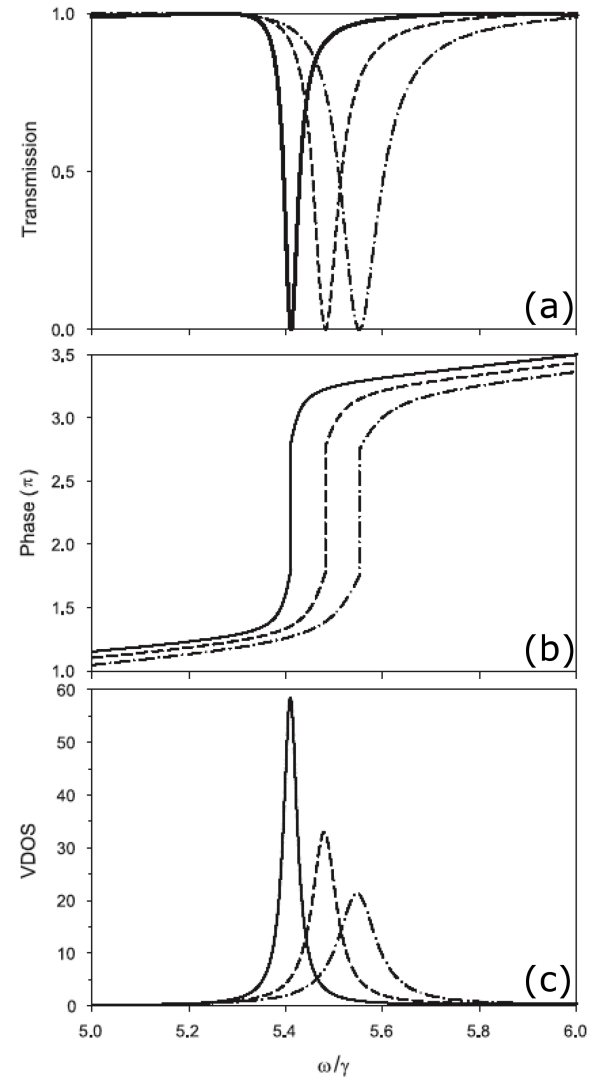

Fig. 4. (a) The same as in Fig. 3a but for three cases of $J$, namely, $J=0.0$ (solid curve), $J=0.05$ (dashed curve) and $J=0.10$ (dashed-dotted curve), respectively. (b) The same as in (a) but for the variation of the phase. (c) The same as in (a) but for the VDOS.

Figure 4a presents the intensity transmission coefficient as a function of $\omega / \gamma$, for the same parameters given in Fig. 3a. The plots are given for $J=0.0$ (redrawn for the sake of comparison), 0.05 and 0.1 , respectively. Two interesting points appear for nonzero values of $J$ : (i) the frequency position of transmission zeros (corresponding to the eigenmodes of the resonator clusters) is shifted to higher/lower frequencies with increasing/decreasing the value of $J$ and (ii) the width of the magnon dips is increased (decreased) with increasing (decreasing) the value of $J$. Here, it is worth mentioning that the second and third dips, corresponding to $J=0.05, J=0.1$, respectively, are due to the hybrid coupling of exchange and dipolar interactions. The shift in the frequency position of transmission zeros is also shown in the plots describing the variation of the phase (Fig. 4b). The VDOS is given in Fig. 4c. For each value of $J$, it shows one peak situated at the frequency for which the modules of $F_{-} g\left(1_{-}, 3_{-}\right)$is zero (see (14)) and the transmission is zero. The system acts as a rejecting filter.

In Fig. 5, we present the state phase $\eta(\omega)$ as a function of frequency for $J=0$. The plot is given for the same parameters considered in Fig. 3a. In the positive frequency range displayed, one can re- 


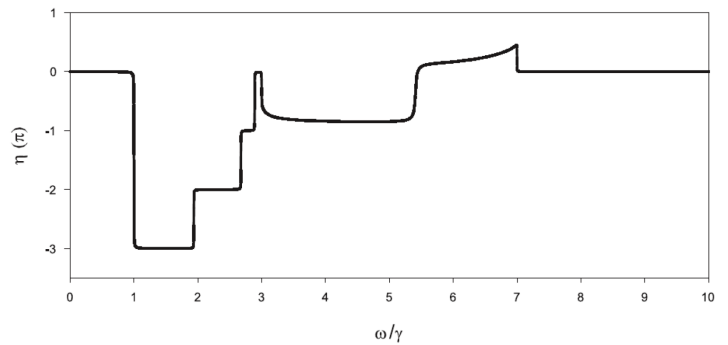

Fig. 5. Variation of the state phase $\eta(\omega)$ as a function of the frequency $\omega / \gamma$, for $J=0$. The other parameters have the same values as in Fig. 3a.

mark the $3 \pi$ phase drop at $(\omega / \gamma)=1$ due to the localized states of the three isolated clusters, the $\pi$ phase jump at all the positions of the localized states and finally the conservation of the number of states. For the negative frequency range, the situation is similar due to the symmetry of the problem.

For $M_{s}^{\prime}=1.3$ and $d_{1}=0.8$, we obtain interesting transmission peaks presented in Fig. 6a. The plots are given for $J=0.0$ (solid curve), 0.1 (dashed curve) and 0.2 (dashed-dotted curve). The effect of the exchange coupling constant is to shift the peak position to higher frequencies. This is also shown in the plots describing the variation of the phase (Fig. 6b). The VDOS is given in Fig. 6c. For each value of $J$, it shows one resonance peak situated at the frequency for which the module of $F_{-} g\left(1_{-}, 3_{-}\right)$is unity (see (14)) and the transmission is unity. The system acts as a selecting filter. These results indicate how the magnon selection frequency may be controlled by selecting the appropriate parameters of the structure.

In Fig. 7, we present the transmission coefficient as a function of $\frac{\omega}{\gamma}$, for the same parameters given in Fig. 3a and several values of $d_{1}$. The six panels clearly show the crossover between the zero of transmission and the total transmission behavior with increasing the distance $d_{1}$.

On the other hand, in Fig. 8 we present the transmission coefficient as a function of $\omega / \gamma$ for fixed $d_{1}$ and several values of the dipole momenta of the additional clusters. Figure 8a shows the behavior of the dips for $d_{1}=1.5$ and $M_{s}^{\prime}=0.9$ (solid curve), 1.0 (dashed curve) and 1.1 (dashed-dotted curve), while Fig. $8 \mathrm{~b}$ shows the behavior of the peaks for $d_{1}=0.8$ and $M_{s}^{\prime}=1.0$ (solid curve), 1.3 (dashed curve) and 1.6 (dashed-dotted curve). One can see that the frequency positions of the peaks (dips) are shifted to a higher value with increasing the value of $M_{s}^{\prime}$. It should be pointed out that in such a structure the zeros of transmission occur when the resonating clusters are weakly coupled to the chain and the frequencies of these zeros can be related to the eigenfrequencies of the isolated adsorbed cluster resonator. On the other hand, the transmission peaks occur when the resonating clusters are strongly coupled to the chain. In that case, the transmission
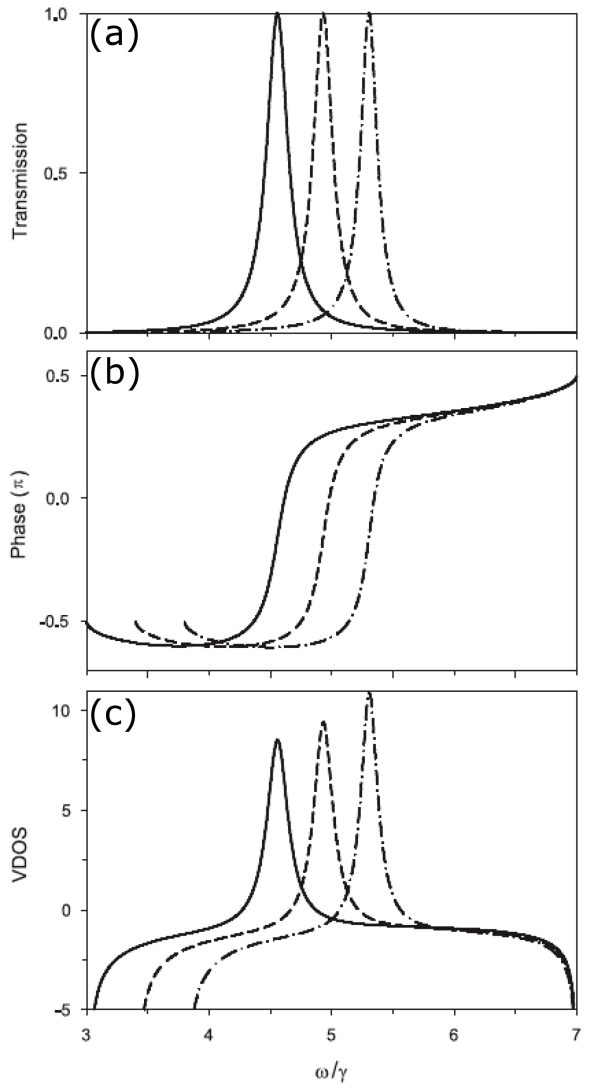

Fig. 6. (a) Transmission as a function of $\omega / \gamma$ for $M_{s}^{\prime}=1.3$, and $d_{1}=0.8$. The other parameters have the same values as in Fig. 3a. The plots are given for $J=0.0$ (solid curve), 0.1 (dashed curve) and 0.2 (dashed-dotted curve), respectively. (b) The same as in (a) but for the variation of the phase. (c) The same as in (a) but for the VDOS.
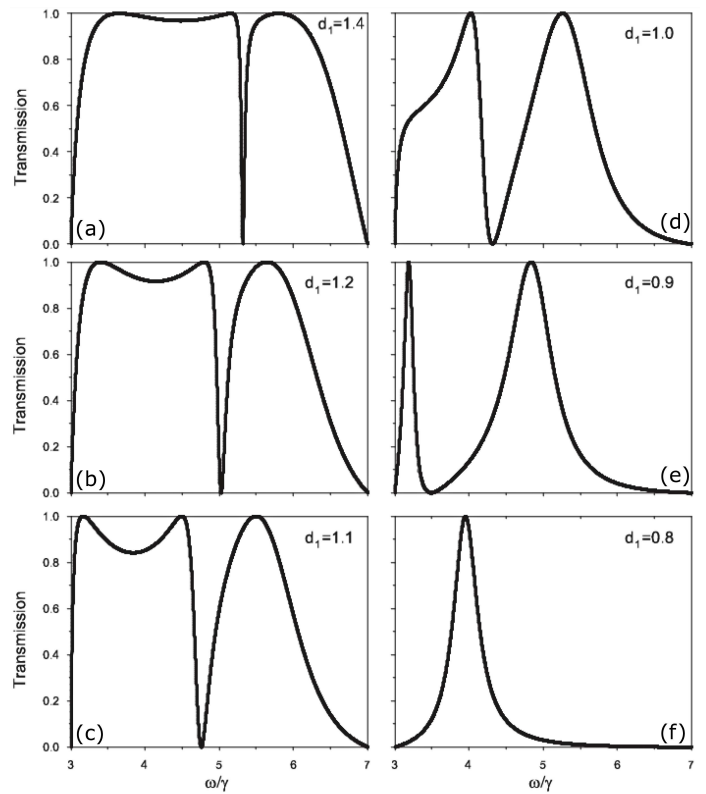

Fig. 7. Transmission as a function of $\omega / \gamma$ for several values of $d_{1}$. The other parameters have the same values as in Fig. 3a. 


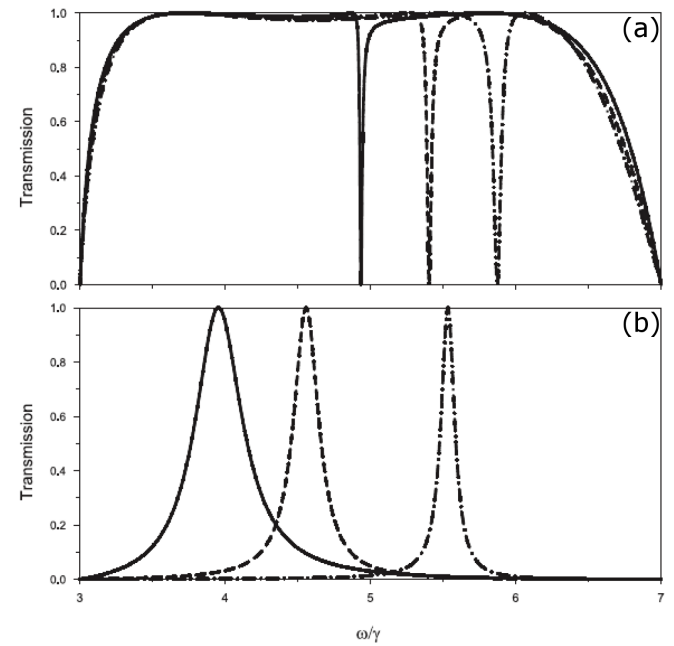

Fig. 8. (a) Transmission as a function of $\omega / \gamma$ for $d_{1}=1.5$ and $M_{s}^{\prime}=0.9$ (solid curve), 1.0 (dashed curve) and 1.1 (dashed-dotted curve), respectively. The other parameters have the same values as in Fig. 3a. (b) The same as in (a) but for $d_{1}=0.8$ and $M_{s}^{\prime}=1.0$, (solid curve), 1.3 (dashed curve) and 1.6 (dashed-dotted curve), respectively.

features can be interpreted as mainly due to the widening of the frequencies falling inside the bulk chain band of the eigenmodes of the ensemble of the perturbed clusters.

Finally, we study the damping effect on the transmission spectra of magnons in the considered resonant structure. The magnetic damping has been probed by measuring the resonance line width in the frequency domain, using the ferromagnetic resonance (FMR) technique [62] that makes accurate predictions about the magnetization dynamics. Two mechanisms are behind the line width FMR: one intrinsic which is related to spin-orbit coupling and magnon-phonon scattering and the other one extrinsic which is due to the presence of inhomogeneities or imperfections within the crystal. In a ferromagnetic system, the spin dynamics is described by using the Landau-Lifshitz equation in which a phenomenological (dimensionless) parameter $\beta$ defines a magnetization relaxation [63]. By taking into consideration the damping effect, the wave vector in (8), (14) and (15) becomes complex:

$$
k=\sqrt{k_{R}+\mathrm{i} k_{I}}
$$

where

$$
k_{R}=\frac{\omega-\gamma\left(H_{0}+6 \frac{M_{s}}{d^{3}}\right)}{\gamma M_{s} d^{2}\left(J-d^{-3}\right)}
$$

and

$$
k_{I}=\beta \frac{\omega}{\gamma M_{s} d^{2}\left(J-d^{-3}\right)}
$$

are the real and imaginary parts of the square of the wave vector.

Figure 9a gives the same results as Fig. 6a but in the presence of damping. We suppose that the considered structure is made of permalloy $(\mathrm{Py})$

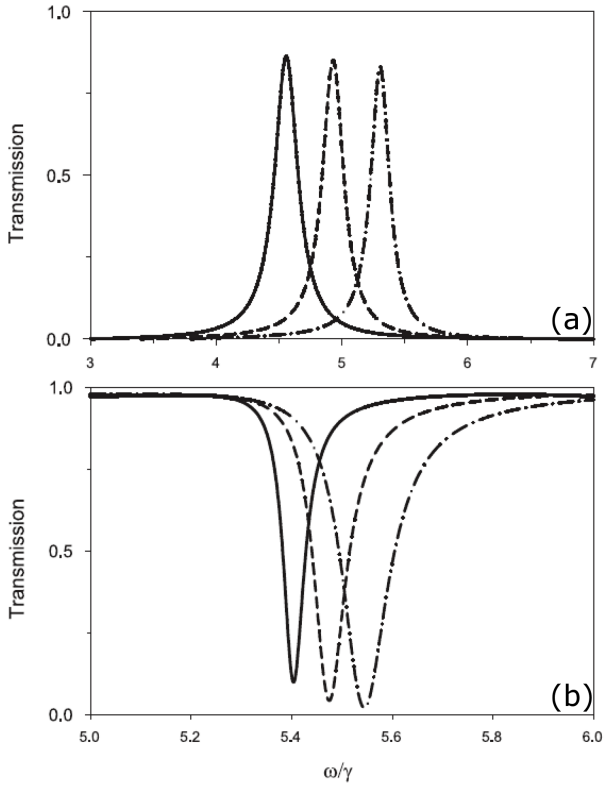

Fig. 9. These figures are similar to Figs. 6a and $4 \mathrm{a}$, respectively, but in the presence of loss in the waveguide.

characterized by the parameter $\beta=0.008$. $\mathrm{Py}$ is the most common material used in magnonics research and offers low damping and isotropic magnetic properties $[5,64,65]$. We can notice that the transmission spectra in Fig. 9a exhibit the same behavior as in Fig. 6a, however, as predicted, the resonances do not reach unity because of the attenuation in the waveguide. On the other hand, the transmission in Fig. 9b (which is the same as in Fig. 4a but in the presence of damping) does not reach zero.

\section{Conclusion}

The objective of this work was to investigate theoretically the effect of the exchange coupling and the attached resonators on magnon propagation in the resonant structure composed of dipole-coupled nanometric magnetic cluster chain and a few additional clusters near the chain. This resonant structure may exhibit peaks and dips in the transmission spectra of magnons depending on the geometrical parameters of the system. The calculated transmission spectrum of magnons in this structure parallels the dispersion relation of the infinite chain. The sharp zeros of transmission are obtained when the resonator clusters are weakly coupled to the chain, while the sharp transmission peaks appear when the resonator clusters are strongly coupled to the chain. The frequency positions of the peaks and dips are shifted to higher (lower) frequencies with increasing/decreasing the value of $J$.

A study of the phase of the transmission function enables us to deduce several properties on the magnon propagation through such structures. For 
the system studied here, the phase of the transmission amplitude exhibits phase jumps around the transmission zeros, whereas the state phase increases monotonically with a fast slope around the resonance frequency. The effect of loss on the transmission of the waveguide in filtering and stopping different frequencies is also discussed. These features have been established through an analysis of the transmission function (amplitude and phase) obtained within the framework of the Green's function technique (a more detailed discussion of the technique is given elsewhere [66]). We try mostly to stress that with the help of nanoclusters, it is possible to construct such a simple nanometric transmission filter for a magnon signal. Furthermore, the closed-form expressions derived in this work enable us to find the optimal parameters for the desired device, enabling one to engineer it at will for specific applications.

At this stage, it is worth to mention once again the conditions of the model's validity. To account for the exchange interaction, we have assumed the size of the clusters to be relatively small (of a few $\mathrm{nm}$ size) and the separation between them should also be in the same range. We have taken into consideration only the nearest-neighbor interactions in our model which obviously fails to be valid in the limit of $d_{1} \gg d$. When $d_{1}$ is of the order of $d$, the positions of the zeros of transmission are also more or less significantly affected by the interaction between the resonator clusters and the infinite linear chain. In other words, the dipole-dipole interactions which were entered to this model are either parallel or perpendicular to the external magnetic field $H_{0}$ and therefore this model avoids the complications due to the anisotropic feature of the dipoledipole interactions.

\section{Acknowledgments}

H. Al-Wahsh and E.H. El Boudouti would like to acknowledge the hospitality of the Institut d'Électronique, de Microélectronique et de Nanotechnologie (IEMN), UMR-CNRS 8520, UFR de Physique, Université de Lille 1.

\section{References}

[1] G. Gubbiotti, R. Silvani, S. Tacchi, M. Madami, G. Carlotti, Z. Yang, A.O. Adeyeye, M. Kostylev, J. Phys. D Appl. Phys. 50, 105002 (2017).

[2] B. Lenk, H. Ulrichs, F. Garbs, M. Münzenberg, Phys. Rep. 507, 107 (2011).

[3] Magnonics: From Fundamentals to Applications, Topics in Applied Physics, Eds. S.O. Demokritov, A.N. Slavin, Springer, Berlin 2013, Vol. 125.

[4] M. Krawczyk, D. Grundler, J. Phys. Condens. Matter 26, 123202 (2014).
[5] A.V. Chumak, A.A. Serga, B. Hillebrands, J. Phys. D Appl. Phys. 50, 244001 (2017).

[6] A.A. Serga, A.V. Chumak, B. Hillebrands, J. Phys. D Appl. Phys. 43, 264002 (2010).

[7] A.V. Chumak, A.A. Serga, B. Hillebrands, Nature Commun. 5, 4700 (2014).

[8] A.V. Chumak, V.I. Vasyuchka, A.A. Serga, B. Hillebrands, Nature Phys. 11, 453 (2014).

[9] A. Akjouj, L. Dobrzynski, H. Al-Wahsh et al., in: Interface Transmission Tutorial Book Series: Magnonics, Elsevier, 2019.

[10] M.A. Morozova, A.Yu. Sharaevskaya, A.V. Sadovnikov, S.V. Grishin, D.V. Romanenko, E.N. Beginin, Yu.P. Sharaevskii, S.A. Nikitov, J. Appl. Phys 120, 223901 (2016).

[11] V.L. Zhang, H.S. Lim, S.C. Ng, M.H. Kuok, X. Zhou, A.O. Adeyeye, AIP Adv. 6, 115106 (2016).

[12] Jun-ichiro Ohe, J. Phys. Soc. Jpn. News Comments 17, 01 (2020).

[13] R.P. Cowburn, D.K. Koltsov, A.O. Adeyeye, M.E. Welland, D.M. Tricker, Phys. Rev. Lett. 83, 1042 (1999).

[14] C.A. Ross, M. Hwang, M. Shima, Phys. Rev. B 65, 144417 (2002).

[15] W. Xu, D.B. Watkins, L.E. Delong, K. Rivkin, J.B. Ketterson, V.V. Metlushko, J. Appl. Phys. 95, 6645 (2004).

[16] A.V. Jausovec, G. Xiong, R.P. Cowburn, Appl. Phys. Lett. 88, 052501 (2006).

[17] J. d'Albuquerque e Castro, D. Altbir, J.C. Retamal, P. Vargas, Phys. Rev. Lett. 88, 237202 (2002).

[18] P. Vargas, D. Altbir, J. d'Albuquerque e Castro, Phys. Rev. B 73, 092417 (2006).

[19] K. Rivkin, A. Heifest, P.R. Sievert, J.B. Ketterson, Phys. Rev. B 70, 184410 (2004).

[20] K. Rivkin, W. Saslow, L.E. De Long, J.B. Ketterson, Phys. Rev. B 75, 174408 (2007).

[21] K. Rivkin, Wentao Xu, L.E. De Long, V.V. Metlushko, B. Ilic, J.B. Ketterson, J. Magn. Magn. Mater. 309, 317 (2007).

[22] A. Khater, M. Abou Ghantous, Surf. Sci. 498, L97 (2002).

[23] M. Abou Ghantous, A. Khater, J. Magn. Magn. Mater. 248, 85 (2002).

[24] R. Huber, D. Grundler, Proc. SPIE 8100, 81000D (2011).

[25] S. Mamica, J.-C.S. Lévy, M. Krawczyk, J. Phys. D Appl. Phys. 47, 015003 (2014). 
[26] R.P. Cowburn, D.K. Koltsov, A.O. Adeyeye, M.E. Welland, D.M. Tricker, Phys. Rev. Lett. 83, 1042 (1999).

[27] A. Lebib, S.P. Li, M. Natali, Y. Chen, J. Appl. Phys. 89, 3892 (2001).

[28] M. Grimsditch, Y. Jaccard, I.K. Schuller, Phys. Rev. B 58, 11539 (1998).

[29] Magnetic Structures of 2D and 3D Nanoparticles: Properties and Applications, Ed. J.-C.S. Levy, CRC Press, 2018.

[30] G. Shimon, A.O. Adeyeye, Appl. Phys. Lett. 109, 032407 (2016).

[31] I.M.L. Billas, J.A. Becker, A. Châtelain, W.A. de Heer, Phys. Rev. Lett. 71, 4067 (1993).

[32] J.T. Lau, A. Fröhlisch, R. Nietubyc, M. Reif, W. Wirth, Phys. Rev. Lett. 89, 057201 (2002).

[33] P. Gambardella, S. Rusponi, M. Veronese et al., Science 300, 1130 (2003).

[34] G. Gubbiotti, M. Conti, G. Carlotti, P. Candeloro, E. Di Fabrizio, K. Guslienko, A. Andre, C. Bayer, A. Slavin, J. Phys. Condens. Matter 16, 7709 (2004).

[35] K. Perzlmaier, M. Buess, C. Back, V. Demidov, B. Hillebrands, S. Demokritov, Phys. Rev. Lett. 94, 057202 (2005).

[36] N. Segreeva, S. Cherif, A. Stashkevich, M. Kostylev, J. Ben Youssef, J. Magn. Magn. Mater. 288, 250 (2005).

[37] L. Giovannini, F. Montoncello, F. Nizzoli, G. Gubbiotti, G. Carlotti, T. Okuno, T. Shinjo, M. Grimsditch, Phys. Rev. B 70, 172404 (2004).

[38] M.G. Cottam, A.A. Maradudin, in: Surface Excitations, Modern Problems in Condensed Matter Sciences, North-Holland, Amsterdam 1984, Vol. 9.

[39] E.L. Albuquerque, M.G. Cottam, Phys. Rep. 376, 225 (2003).

[40] J.P. Nguenang, A.J. Kenfack, T.C. Kofane, Phys. Rev. E 66, 056613 (2002).

[41] H. Al-Wahsh, L. Dobrzynski, B. DjafariRouhani, G. Hernández-Cocoletzi, A. Akjouj, Surf. Sci. 600, 4883 (2006).

[42] H. Al-Wahsh, L. Dobrzynski, B. DjafariRouhani, A. Akjouj, Surf. Sci. 601, 4801 (2007).

[43] H. Al-Wahsh, B. Djafari-Rouhani, L. Dobrzynski, A. Akjouj, Surf. Sci. 602, 1795 (2008).

[44] E.M. Lifshitz, L.P. Pitaevskii, Statistical Physics, Pergamon Press, New York 1980, Part II, Sect. 69.
[45] L.R. Walker, in: Magnetism, Eds, G.T. Rado, H. Suhl, Academic, New York 1963, Vol. 1, Ch. 8, p. 299.

[46] D.L. Mills, A.A. Maradudin, J. Phys. Chem. Solids 28, 1855 (1967).

[47] L. Dobrzynski, Surf. Sci. Rep. 11, 139 (1990).

[48] L. Dobrzynski, V.R. Velasco, F. GarciaMoliner, Phys. Rev. B 35, 5872 (1987).

[49] K. Rivkin, L.E. De Long, J.B. Ketterson, J. Appl. Phys. 97, 10E309 (2005).

[50] I. Rotter, A.F. Sadreev, Phys. Rev. E 71 , 046204 (2005).

[51] H. Barkay, E. Narevicius, N. Moiseyev, Phys. Rev. B 67, 045322 (2003).

[52] L. Dobrzynski, A. Akjouj, E. El Boudouti et al., in: Interface Transmission Tutorial Book Series: Phononics, Elsevier, 2017.

[53] T. Taniguchi, M. Buttiker, Phys. Rev. B 60, 13814 (1999).

[54] J. Friedel, Philos. Mag. 43, 153 (1952).

[55] H.W. Lee, Phys. Rev. Lett. 82, 2358 (1999).

[56] H. Al-Wahsh, E.H. El Boudouti, B. Djafari-Rouhani, A. Akjouj, L. Dobrzynski, Phys. Rev. B 75, 125313 (2007).

[57] A. Yacoby, M. Heiblum, D. Mahalu, H. Shtrikman, Phys. Rev. Lett. 74, 4047 (1995).

[58] R. Schuster, E. Buks, M. Heiblum, D. Mahalu, V. Umansky, H. Shtrikman, Nature (London) 385, 417 (1997).

[59] A. Mouadili, E.H. El Boudouti, A. Soltani, A. Talbi, A. Akjouj, B. Djafari-Rouhani, J. Appl. Phys. 113, 164101 (2013).

[60] A. Mouadili, E.H. El Boudouti, A. Soltani, A. Talbi, A. Akjouj, B. Djafari-Rouhani, J. Phys. Condens. Matter 26, 505901 (2014).

[61] J.O. Vasseur, A. Akjouj, L. Dobrzynski, B. Djafari-Rouhani, E.H. El Boudouti, Surf. Sci. Rep. 54, 1 (2004).

[62] C. Kittel, Phys. Rev. 73, 155 (1948).

[63] A. Barman, J. Sinha, Spin Dynamics and Damping in Ferromagnetic Thin Films and Nanostructures, Springer Nature, 2018.

[64] D.J. Twisselmann, R.D. McMichael, J. Appl. Phys. 93, 6903 (2003).

[65] S.S. Kalarickal, P. Krivosik, M. Wu, C.E. Patton, J. Appl. Phys. 99, 093909 (2006).

[66] H. Al-Wahsh, A. Akjouj, B. DjafariRouhani, L. Dobrzynski, Surf. Sci. Rep. 66, 29 (2011). 